\title{
Effect of different jump distributions on the dynamics of jump processes
}

\author{
Edoardo Daly* \\ Department of Civil Engineering, Monash University, Clayton, Victoria, Australia \\ Amilcare Porporato ${ }^{\dagger}$ \\ Department of Civil and Environmental Engineering, Duke University, Durham, North Carolina, 27708-0287 USA
}

(Received 1 January 2010; published 28 June 2010)

\begin{abstract}
The paper investigates stochastic processes forced by independent and identically distributed jumps occurring according to a Poisson process. The impact of different distributions of the jump amplitudes are analyzed for processes with linear drift. Exact expressions of the probability density functions are derived when jump amplitudes are distributed as exponential, gamma, and mixture of exponential distributions for both natural and reflecting boundary conditions. The mean level-crossing properties are studied in relation to the different jump amplitudes. As an example of application of the previous theoretical derivations, the role of different rainfalldepth distributions on an existing stochastic soil water balance model is analyzed. It is shown how the shape of distribution of daily rainfall depths plays a more relevant role on the soil moisture probability distribution as the rainfall frequency decreases, as predicted by future climatic scenarios.
\end{abstract}

DOI: 10.1103/PhysRevE.81.061133

PACS number(s): 02.50.Ey, 92.40.Oj, 92.40.We

\section{INTRODUCTION}

Dynamical systems with trajectories forced by instantaneous discontinuous transitions (i.e., jumps) are of interest in various disciplines, such as queuing theory, with applications to storage problems, insurance and stock market modeling [1-3], and biophysics, where jump processes have been adopted in neuron models to describe the voltage across a nerve membrane [4-7]. They have been also used in ecology to model, e.g., drastic fluctuations in population dynamics $[8-10]$ and the occurrence of fires in ecosystems $[11,12]$. Further applications can be found in hydrology, e.g., to generate stream flow data [13] and describe the soil water balance [14], in the study of noise-driven transport [15] and noise-induced synchronization [16-18], in models of human action executions $[7,19]$, and in the physics of solar flare rates $[20,21]$.

Many of the dynamical systems previously mentioned may be described in terms of a single representative stochastic variable $x(t)$, which follows a deterministic trajectory perturbed by instantaneous jumps of random amplitudes occurring according to a Poisson process. In the following we will focus on systems for which the dynamics of the random variable $x(t)$ is driven by the stochastic differential equation

$$
\frac{d x}{d t}=-f(x)+F(x, t)
$$

where $f(x)$ is a drift that drives the deterministic behavior of the system between jumps and $F(x, t)$ is a Poisson process, defined as

\footnotetext{
*edoardo.daly@eng.monash.edu.au

†amilcare@duke.edu
}

$$
F(x, t)=\sum_{n=1}^{N(t)} y_{n} \delta\left(t-t_{n}\right)
$$

where the sequence $\left\{t_{n}\right\}(n=1,2, \ldots)$ represents the instants of occurrence of $n$ events of the Poisson counting process $N(t)(t \geq 0)$ with intensity $\lambda$, which will be considered constant, and $\left\{y_{n}\right\}$ is a sequence of independent random variables, identically distributed as $h(y)$ and independent of the Poisson process $N(t)$.

The emphasis of this study is on the probability density function (pdf) of the state variable $x(t), p(x, t)$. We will analyze how $p(x, t)$ of processes with the same drift and forced by jumps occurring at the same rate $\lambda$ are shaped by different jump distributions $h(y)$. The analysis is extended to systems bounded by reflecting barriers, and the obtained results will be used to study the effect of different jump distributions on the time of crossing of a constant level. As an application of these theoretical results, we discuss a stochastic model for the soil water balance in arid and semiarid ecosystems, extending the work of [22] to analyze the role of different forms of rainfall-depth distributions in the stochastic soil water balance equation.

\section{GENERAL PROPERTIES}

\section{A. Naturally bounded processes}

According to Eq. (1), the transition probability density function of $x(t), p(x, t)$, satisfies the forward master equation [23]

$$
\frac{\partial}{\partial t} p(x, t)=\frac{\partial}{\partial x}[f(x) p(x, t)]-\lambda p(x, t)+\lambda \int_{-\infty}^{+\infty} p(x-z, t) h(z) d z,
$$

where the terms on the right-hand side are, respectively, the contributions to $p(x, t) d x$ due to the drift $f(x)$, the loss of probability generated by jumps that make the process leave 


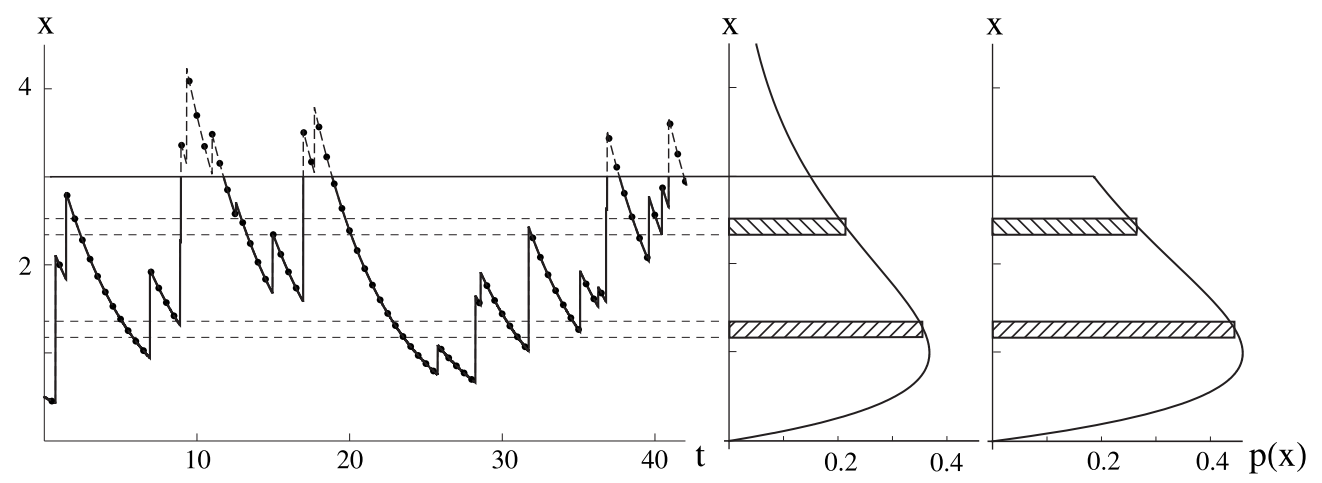

FIG. 1. Example of time series and effect of a reflecting barrier.

the present trajectory, and the contribution to the probability due to jumps to the state $x$. The initial condition to solve Eq. (3) will be assumed to be an atom of probability in $x=x(0)$ $=x_{0}$, i.e., $p(x, 0)=\delta\left(x-x_{0}\right)$.

For linear drift (see, e.g., Sec. III), a convenient approach to solve Eq. (3) and to derive the statistical properties of $x(t)$ is through the two-sided Laplace transform

$$
p^{*}(u, t)=\int_{-\infty}^{+\infty} e^{-u x} p(x, t) d x
$$

with initial condition $p^{*}(u, 0)=\exp \left(-u x_{0}\right)$. The Laplace transform can be used to calculate the moments of $x$ whereas the inversion of $p^{*}(u, t)$ was not possible.

An application of the use of the Laplace transform to solve Eq. (3) can be derived for jump processes in the absence of drift, i.e., the Poisson process. When $f(x)=0$, applying the Laplace transform to Eq. (3) yields

$$
p_{x}^{*}(u, t)=e^{-\lambda t\left[1-h^{*}(u)\right]},
$$

where it has been assumed that $x_{0}=0$. The mean and variance of $x$ change in time as $\mu_{x}=\lambda \mu_{y} t$ and $\sigma_{x}^{2}=\kappa_{2}=\lambda \sigma_{y}^{2} t$, with $\mu_{y}$ and $\sigma_{y}^{2}$ as the mean and variance of the jumps, respectively. By normalizing $x$ as $z=\left(x-\mu_{x}\right) / \sigma_{x}$, one can write

$$
p_{z}^{*}(u, t)=e^{u \mu_{x} / \sigma_{x}} p_{x}^{*}\left(u / \sigma_{x}\right)=e^{u \mu_{x} / \sigma_{x}} e^{-\lambda t\left[1-h^{*}\left(u / \sigma_{x}\right)\right]} .
$$

For $u \rightarrow 0, h^{*}\left(u / \sigma_{x}\right)$ can be approximated as

$$
\begin{aligned}
\left.h^{*}\left(u / \sigma_{x}\right)\right|_{u \rightarrow 0}= & h^{*}(0)+\left.\frac{d}{d s} h^{*}(s)\right|_{s=0} \frac{u}{\sigma_{x}}+\left.\frac{d^{2}}{d s^{2}} h^{*}(s)\right|_{s=0} \frac{u^{2}}{2 \sigma_{x}^{2}} \\
& +\cdots \\
= & 1-\mu_{y} \frac{u}{\sigma_{x}}+E\left[y^{2}\right] \frac{u^{2}}{2 \sigma_{x}^{2}}+\cdots
\end{aligned}
$$

Inserting the previous equation into Eq. (6) and taking the limit for $t \rightarrow+\infty$ yields $p_{z}^{*}(u)=\exp \left(u^{2} / 2\right)$, which means that $z$ tends to a normal distribution, as expected from the central limit theorem.

An exact derivation of this application of the central limit theorem can be obtained for jumps exponentially distributed, for which $p_{x}^{*}(u, t)$ can be inverted to give (see, for example, [24])

$$
p(x, t)=\exp (-x \gamma-\lambda t)\left(\sqrt{\frac{\lambda \gamma t}{x}} I_{1}(2 \sqrt{\lambda \gamma x t})+\delta(x)\right),
$$

where $\gamma$ is the parameter of the exponential distribution of the jumps and $I_{\nu}(\cdot)$ is the modified Bessel function of the first kind of order $\nu[25]$.

\section{B. Reflecting boundaries}

In the presence of reflecting barriers, obtaining the solution $p(x, t)$ becomes more difficult. However, if the system reaches steady-state conditions [i.e., the pdf of $x(t)$ does not vary in time], the solution of Eq. (3) for a bounded system is simply related to that of the same system with natural boundaries by means of a rescaling within its domain of existence [14] (note, however, that it is not possible to obtain a general form of the steady-state pdf for generic jump distributions). This is schematically shown in Fig. 1 for a process with a natural bound at $x=0$.

For the sake of simplicity, let us start by imagining sampling a trajectory at equal time intervals for a long period of time. To estimate the frequency distribution we first build a histogram with the height of each rectangle proportional to the number of sampled points in each bin. The frequency distribution can be estimated by dividing the height of each rectangle by the total number of sampled points. When a reflecting barrier is introduced at $x=x_{b}$ (i.e., $\left.x \in\left(0, x_{b}\right]\right)$, the portions of trajectories above the bound no longer exist, and whenever a jump generates an overshoot the system starts from $x_{b}$. As shown in Fig. 1, the number of points in each bin $\Delta x$ below the barrier is the same for the bounded and unbounded processes. Therefore, the histogram of the bounded process coincides with the histogram of the unbounded process for $x \leq x_{b}$. However, the total number of points sampled in the unbounded process is larger than that of the bounded process because of the portions of trajectories above $x_{b}$. Accordingly, the frequency distribution of the two processes needs to be scaled differently. The bounded process is scaled using only the number of points below the threshold, while the unbounded process needs to be scaled using all the sampled points.

This reasoning can be extended to a continuous process. Assuming that the pdf of the unbounded process [i.e., $x$ $\in(0,+\infty)]$ is known, the area subtended by such a pdf above two intervals $\Delta x_{1}$ and $\Delta x_{2}$ represents the percentage of time 
that the process spends in these two intervals, respectively. When a reflecting barrier is added, the percentage of time spent by the bounded process in the intervals $\Delta x_{1}$ and $\Delta x_{2}$ is higher than in the unbounded case. However, because the dynamics between jumps follow the same deterministic law defined by the drift $f(x)$, the ratio between the shaded areas in the two pdf's in Fig. 1 must be the same for the bounded and unbounded cases. The pdf of the bounded process can thus be calculated by rescaling the pdf of the unbounded process, so that the area of the pdf equals 1 . As a result, the pdf $p_{b}(x)$ of a process bounded between $-\infty$ and $x_{b}$ is proportional to the pdf $p_{u}(x)$ of the corresponding unbounded process within the same interval $\left[p_{b}(x)=C p_{u}(x)\right]$. Because

$$
\int_{-\infty}^{x_{b}} p_{b}(x) d x=C \int_{-\infty}^{x_{b}} p_{u}(x) d x=1,
$$

$C=1 / \int_{-\infty}^{x_{b}} p_{u}(x) d x$.

The same procedure, with appropriate modifications of the extremes of integrations, can be used when the system is bounded between two reflecting barriers and in the presence of atoms of probability. This would also apply if the system was additionally forced by white Gaussian noise, as long as the process has reached steady-state conditions [24,26].

\section{Level-crossing properties}

In various practical applications, it is important to have information about the time that a stochastic variable at steady state spends above (or below) a certain threshold. An exact complete characterization of the statistics of such a time is only available for a few stochastic processes [27]. However, in the case of jump processes general results can be derived for the mean duration of an excursion above (or below) a threshold when jumps and drift are both either positive or negative.

In the case of always positive jumps, if $f(x)$ is positive, the dynamics of $x$ is due to two opposite mechanisms [see Eq. (1)]. The drift makes $x$ decrease and the positive jumps force $x$ to increase. When none of these two mechanisms prevails, $x(t)$ can reach steady-state conditions. At steady state, if $\nu_{\xi}$ is the average frequency of up-crossings of a level $\xi$ of $x(t)$, the average duration of time intervals during which $x(t)<\xi$ is $[27-29]$

$$
T_{\xi^{-}}=\frac{P(\xi)}{\nu_{\xi}},
$$

where $P(\xi)$ is the probability that $x(t)<\xi$, which represents the average length of time spent by the process below $\xi$ per unit time. Since an up-crossing can only be caused by a jump, the frequency of up-crossing $\nu_{\xi}$ can be calculated as follows. By definition, $\nu_{\xi} d t$ is the probability of having an up-crossing of $\xi$ in the interval $d t$. Because of the independence between jump occurrence and amplitude, $\nu_{\xi} d t$ is given by the product between the probability of occurrence of a jump, $\lambda d t$, and the probability that such a jump moves $x$ above $\xi$. The probability that a jump produces an up-crossing of $\xi$ starting from a level $z<\xi$ is

$$
\int_{\xi-z}^{+\infty} h(y) d y .
$$

Therefore, the probability that a jump causes an up-crossing starting from any $z<\xi$ can be written as

$$
\int_{-\infty}^{\xi}\left(\int_{\xi-z}^{+\infty} h(y) d y\right) p(z) d z,
$$

from which

$$
\nu_{\xi}=\lambda \int_{-\infty}^{\xi}\left(\int_{\xi-z}^{+\infty} h(y) d y\right) p(z) d z
$$

Using an extension of the Rice formula, the frequency $\nu_{\xi}$ can be also calculated as [28-31],

$$
\nu_{\xi}=f(\xi) p(\xi),
$$

which represents the frequency of down-crossing. In fact, as derived in Appendix A, the integration of Eq. (3) in steadystate conditions simply states that the frequency of upcrossing equals that of down-crossing.

As already noted by [29], the introduction of a reflecting barrier above the level $\xi$ (i.e., $x_{b}>\xi$ ) does not modify the average duration of an excursion below $\xi$, because $p_{b}(x)$ $=C p_{u}(x)$. As the probability of being below $\xi$ increases for $x_{b}$ becoming closer to $\xi$, so does the frequency of up-crossing, thereby maintaining $T_{\xi^{-}}$constant. Therefore, the dynamics of the process above $\xi$ do not affect the value of $T_{\xi^{-}}$. On the other hand, as intuitively expected, the presence of a bound at $x_{b}>\xi$ becomes important in determining the average duration of an excursion above $\xi$, i.e.,

$$
T_{\xi^{+}}=\frac{1-P(\xi)}{\nu_{\xi}}=\frac{\int_{\xi}^{x_{b}} p_{b}(u) d u}{\lambda \int_{-\infty}^{\xi}\left(\int_{\xi-u}^{+\infty} h(y) d y\right) p_{b}(u) d u} .
$$

\section{LINEAR DRIFT}

In this section we compare a few systems with the same linear drift $f(x)=k x(k>0)$ and forced by a compound Poisson process with constant rate $\lambda$ and different distributions of the jump amplitudes, $h(y)$. These types of systems in engineering applications are commonly called filtered Poisson processes with exponential shape function, while in physics these are known as shot-noise or generalized OrnsteinUhlenbeck processes (see Ref. [32]). We note in passing that the case of linear deterministic decays between jumps (i.e., constant drift) with threshold at zero has been discussed in depth in the seminal work of Takács [33].

The shot-noise process has a natural bound at $x=0$, so that in the unbounded case its domain is the positive axis. Because $x \geq 0$, in the unbounded case the Laplace transform of $p(x, t)$ reads (see Appendix $\mathrm{B})$ 


$$
p^{*}(u, t)=e^{-x_{0} u e^{-k t}} \exp \left(-\lambda \int_{u e^{-k t}}^{u} \frac{\left[1-h^{*}\left(u^{\prime}\right)\right]}{k u^{\prime}} d u^{\prime}\right) .
$$

The pdf $p(x, t)$ can thus be derived by inverting the Laplace transform. In most of the cases the inversion is not possible and it is more convenient to operate directly on the Laplace transform in steady-state conditions, i.e.,

$$
p^{*}(u)=\exp \left(-\lambda \int_{0}^{u} \frac{1-h^{*}\left(u^{\prime}\right)}{k u^{\prime}} d u^{\prime}\right)
$$

Accordingly, the mean of the process is

$$
\mu_{x}=x_{0} e^{-k t}+\frac{\mu_{y} \lambda}{k}\left(1-e^{-k t}\right) .
$$

Higher-order moments of $x$ can be calculated from the cumulants, which read [1]

$$
\kappa_{n}=E\left[y^{n}\right] \frac{\lambda}{n k}\left(1-e^{-n k t}\right),
$$

where $E\left[y^{n}\right]=\int_{-\infty}^{+\infty} y^{n} h(y) d y$.

In what follows, cases with different distributions $h(y)$ are analyzed, assuming that the jumps are positive [i.e., $h(y)=0$ for $y<0]$. We note that exact steady-state pdf's of $x$ can be calculated also for some distributions with both positive and negative jumps, such as, e.g., the two-sided exponential distribution, $h(y)=(r / 2) \exp (-r|y|)$, for which $p(x)$ is a so-called $K$ distribution [26,34],

$$
p(x)=\sqrt{\frac{2}{\pi}} \frac{r(r|x|)^{s-1 / 2}}{2^{s} \Gamma(s)} K_{s-1 / 2}(r|x|),
$$

with $s=\lambda /(2 k), \Gamma(\cdot)$ as the gamma function, and $K_{\nu}(\cdot)$ as the modified Bessel function of the second kind [25].

\section{A. Exponential distribution}

The case with $h(y)=\gamma \exp (-\gamma y)$ is well known in the literature and it is briefly discussed here for completeness. The Laplace transform can be derived as

$$
p_{e}^{*}(u, t)=e^{-x_{0} u e^{-k t}}\left(\frac{\gamma+u e^{-k t}}{\gamma+u}\right)^{\lambda / k},
$$

and can be inverted to obtain [35-37]

$$
\begin{aligned}
p_{e}(x, t)= & \Theta\left(x-x_{0} e^{-k t}\right)\left\{e^{-\lambda t} \delta\left(x-x_{0} e^{-k t}\right)\right. \\
& +e^{-\lambda t-\gamma\left(x-x_{0} e^{-k t}\right)} \frac{\lambda \gamma}{k}\left(e^{k t}-1\right) \\
& \left.\times{ }_{1} F_{1}\left[1-\lambda / k, 2, \gamma\left(x-x_{0} e^{-k t}\right)\left(1-e^{k t}\right)\right]\right\},
\end{aligned}
$$

where $\Theta(\cdot)$ is the Heaviside function and ${ }_{1} F_{1}(\cdot, \cdot, \cdot)$ is the Kummer confluent hypergeometric function [25]. In steadystate condition, Eq. (22) becomes the gamma distribution

$$
p_{e}(x)=\frac{\gamma^{\lambda / k}}{\Gamma(\lambda / k)} e^{-\gamma x} x^{\lambda / k-1} .
$$

The average duration of an excursion below a level $\xi$ can be exactly calculated as (see also Refs. $[29,32,36,38]$ )

$$
T_{\xi^{-}}(\xi)=\frac{1}{\lambda}{ }_{1} F_{1}\left(1,1+\frac{\lambda}{k}, \gamma \xi\right) .
$$

We recall that in steady-state conditions, when the jumps are exponentially distributed, solutions for processes with generic forms of the drift can be obtained by solving Eq. (3) as [14]

$$
p(x)=\frac{N}{f(x)} \exp \left(-\gamma x+\lambda \int_{x} \frac{d u}{f(u)}\right),
$$

with $N$ as a normalization constant to assure that $\int_{0}^{+\infty} p(x) d x$ $=1$.

\section{B. Gamma distribution}

When the jump amplitudes are distributed as $h(y)$ $=\theta^{\alpha} y^{\alpha-1} e^{-\theta y} / \Gamma(\alpha)$, a general solution for the Laplace transform of the pdf of $x(t)$ can be derived as shown in Appendix C to obtain

$$
\begin{aligned}
& p_{g}^{*}(u, t)= e^{-x_{0} u e^{-k t}-\lambda t} \\
& \times \frac{\exp \left\{\frac{\lambda}{k \alpha}\left[\theta /\left(u e^{-k t}\right)\right]^{\alpha} F\left(\alpha, \alpha ; \alpha+1 ;-\theta /\left[u e^{-k t}\right]\right)\right\}}{\exp \left[\frac{\lambda}{k \alpha}(\theta / u)^{\alpha} F(\alpha, \alpha ; \alpha+1 ;-\theta / u)\right]},
\end{aligned}
$$

where $F(\cdot, \cdot ; \cdot ; \cdot)$ is the hypergeometric function [25]. When the system reaches steady state, the pdf becomes [25]

$$
p_{g}^{*}(u)=N(\theta / u)^{\lambda / k} \exp \left[-\frac{\lambda}{k \alpha}(\theta / u)^{\alpha} F(\alpha, \alpha ; \alpha+1 ;-\theta / u)\right],
$$

where $N$ is a normalization constant such that $p_{g}^{*}(0)=1$.

An exact distribution in steady-state conditions can be obtained when the shape factor $\alpha$ is equal to 2, i.e., $h(y)$ $=\theta^{2} y e^{-\theta y} / \Gamma(2)$. In this case,

$$
p_{g}^{*}(u)=f_{1}^{*}(u) f_{2}^{*}(u)=\exp \left(-\frac{\lambda u}{k(\theta+u)}\right)\left(\frac{\theta}{\theta+u}\right)^{\lambda / k} .
$$

The two functions $f_{1}^{*}(u)$ and $f_{2}^{*}(u)$ can be inverted separately as [35]

$$
\begin{gathered}
f_{1}(x)=\frac{\theta^{\lambda / k}}{\Gamma(\lambda / k)} e^{-\theta x} x^{\lambda / k-1}, \\
f_{2}(x)=e^{\lambda / k-\theta x} \sqrt{\frac{\lambda \theta}{k x}} I_{1}\left(2 \sqrt{\frac{\lambda \theta x}{k}}\right)+e^{\lambda / k-\theta x} \delta(x),
\end{gathered}
$$

where $f_{1}(x)$ is a gamma distribution and $I_{\nu}(\cdot)$ is the modified Bessel function of the first kind [25]. The pdf $p(x)$ can be 
evaluated as the convolution between $f_{1}(x)$ and $f_{2}(x)$, i.e., $p_{g}(x)=\int_{0}^{x} f_{1}(x-z) f_{2}(z) d z$, and reads

$$
p_{g}(x)=e^{-\lambda / k-\theta x}(k x / \lambda)^{(\lambda / k-1) / 2} \theta^{(1+\lambda / k) / 2} I_{\lambda / k-1}[2 \sqrt{\theta \lambda x / k}] .
$$

\section{Mixture of exponential distributions}

Mixtures of exponential distributions (or hyperexponential distributions) find applications in queuing and communication problems, and they have been efficiently used to approximate heavy-tail distributions, such as Pareto and Weibull distributions [39]. A mixture of $m$ exponential distributions has the form

$$
h(y)=\sum_{i=1}^{m} \omega_{i} \gamma_{i} e^{-\gamma_{i} y},
$$

with $\omega_{i} \in(0,1)$ and $\sum_{i=1}^{m} \omega_{i}=1$. By using Eq. (16), the Laplace transform of the pdf of $x$ can be obtained as

$$
p_{m}^{*}(u, t)=e^{-x_{0} u e^{-k t}} \prod_{i=1}^{m}\left(\frac{\gamma_{i}+u e^{-k t}}{\gamma_{i}+u}\right)^{\lambda \omega_{i} / k},
$$

which implies that $p_{m}(x, t)$ is given by the convolution of $f_{0}=\delta\left(x-x_{0} e^{-k t}\right)$ and $m$ functions $f_{i}(i=1,2, \ldots, m)$, each of which reads

$$
\begin{aligned}
f_{i}(x, t)= & e^{-\lambda \omega_{i} t} \delta(x)+e^{-\lambda \omega_{i} t-\gamma_{i} x} \frac{\lambda \gamma_{i}}{k}\left(e^{k t}-1\right)_{1} F_{1} \\
& \times\left[1-\lambda \omega_{i} / k, 2, \gamma_{i} x\left(1-e^{k t}\right)\right] .
\end{aligned}
$$

The steady-state pdf of $x$ is given by the convolution of $m$ gamma distributions,

$$
p_{i}(x)=\frac{\gamma_{i}^{\lambda \omega_{i} / k}}{\Gamma\left(\lambda \omega_{i} / k\right)} e^{-\gamma_{i}^{x} x x^{\lambda \omega_{i} / k-1}} .
$$

In the particular case of a mixture of two exponential distributions, $w_{2}$ equals $1-w_{1}$ and the steady-state pdf of $x$ can be derived as (see Appendix D)

$$
\begin{aligned}
p_{m}(x)= & \frac{e^{-\gamma_{1} x} \gamma_{1}^{\lambda \omega_{1} / k} \gamma_{2}^{\lambda\left(1-\omega_{1}\right) / k} x^{-1+\lambda / k}}{\Gamma(\lambda / k)} \\
& \times_{1} F_{1}\left(\frac{\lambda}{k}\left(1-\omega_{1}\right), \frac{\lambda}{k},\left(\gamma_{1}-\gamma_{2}\right) x\right) .
\end{aligned}
$$

\section{Comparison between processes with different jump distributions}

A comparison among processes that only differ in the distribution of the jump amplitudes (i.e., with the same $\lambda, k$, and $x_{0}$ ) shows how the jumps shape the pdf of $x$. According to Eqs. (18) and (19), the moments of $x$ strongly depend on the moments of the distribution of the jump sizes. Different distributions of the jumps with the same mean lead to the same mean for $x$, while higher-order moments are different, as it can be seen from the cumulants. If we compare $\kappa_{2}$ (i.e., the

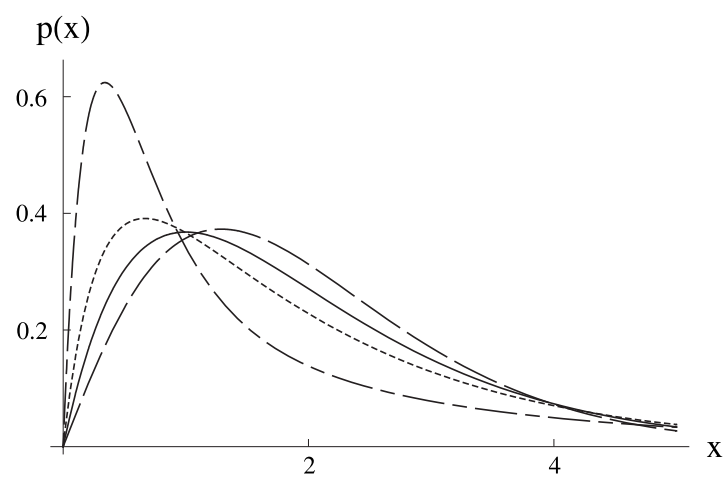

FIG. 2. Comparison between steady-state $p(x)$ of processes with the same mean $(x=2)$ forced by jumps with different distributions: exponential (continuous line: $\gamma=1$ ), gamma (dashed line: $\alpha=2, \theta$ $=2$ ), and mixture of two exponential distributions (dotted line: $\omega_{1}$ $=0.4, \gamma_{1}=4, \gamma_{2}=2 / 3$; dashed-dotted line: $\left.\omega_{1}=0.8, \gamma_{1}=4, \gamma_{2}=1 / 4\right)$. Common parameters are $k=0.2$ and $\lambda=0.4$.

variance $\sigma^{2}$ ) when the system is forced by jumps exponentially and gamma distributed, the difference between the variances of the two processes results to

$$
\sigma_{e}^{2}(t)-\sigma_{g}^{2}(t)=\frac{1}{\gamma^{2}}\left(1-\frac{1}{\alpha}\right) \frac{\lambda}{2 k}\left(1-e^{-2 k t}\right) .
$$

As expected the difference between the two variances increases in time and reaches its maximum value at steady state. Higher moments can be also evaluated. For example, the differences between the skewness and kurtosis coefficients at steady state are $2-2 / \sqrt{\alpha}$ and $6-6 / \alpha$, respectively. When $\alpha=1$, the gamma distribution becomes exponential and the moments of two processes coincide. As $\alpha$ grows the difference between the two processes increases. Larger differences can be obtained using a mixture of exponential distributions because the higher number of parameters permits more flexibility.

Examples of these differences at steady state are shown in Fig. 2. Although the mean of $x$ in steady-state conditions is the same for the four processes because $\mu_{y}$ is the same for all the jump distributions, the higher-order statistics appear very different. Jumps gamma distributed generate modes of $p_{g}(x)$ that are larger than the modes of $p_{e}(x)$ and $p_{m}(x)$. With an opportune choice of the parameters, $p_{m}(x)$ can present a peak for low values of $x$ and a tail higher than the other distributions.

These differences in the statistics induced by the various distributions of the jump amplitudes are reflected in the crossing properties. Figure 3 reports the average duration of excursion below a level $\xi$ and the frequency of up-crossing (or down-crossing) the same level as a function of $\xi$ itself. As $\xi$ increases, $T_{\xi^{-}}$becomes larger for all the distributions. Differences in $T_{\xi^{-}}$become more marked for larger values of $\xi$. Mixtures of exponential distributions, which present heavier tails, allow the systems to reach higher levels more often (i.e., $\nu_{\xi}$ is larger), thereby sensibly reducing $T_{\xi^{-}}$.

\section{SOIL WATER BALANCE}

The temporal evolution of the soil water content controls the dynamics of vegetation and soil biogeochemistry as well 

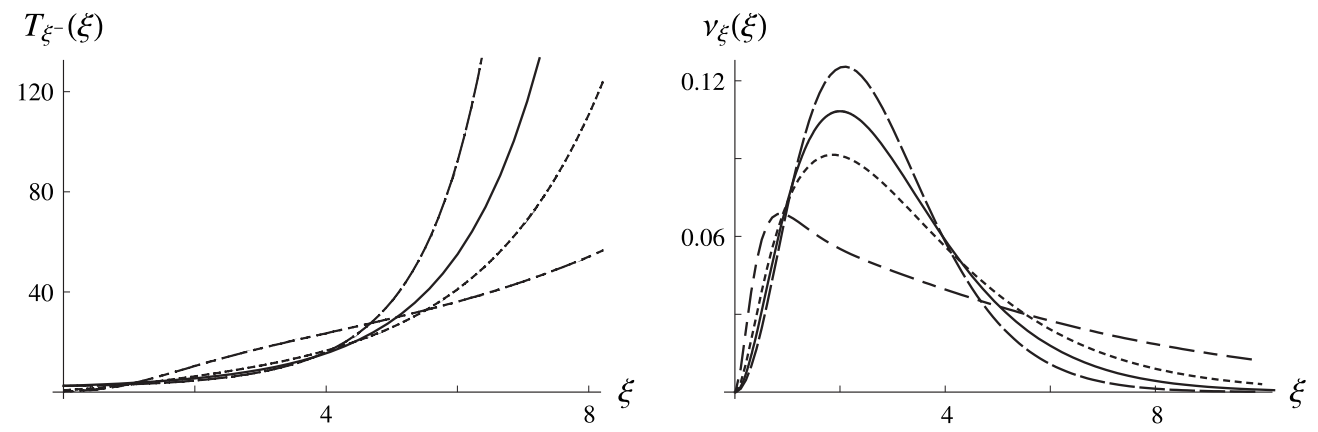

FIG. 3. Comparison between the average duration of an excursion below a level $\xi$ and frequency of up-crossing a level $\xi$ for processes forced by jumps with different distributions: exponential (continuous line: $\gamma=1$ ), gamma (dashed line: $\alpha=2, \theta=2$ ), and mixture of two exponential distributions (dotted line: $\omega_{1}=0.4, \gamma_{1}=4, \gamma_{2}=2 / 3$; dashed-dotted line: $\omega_{1}=0.8, \gamma_{1}=4, \gamma_{2}=1 / 4$ ). Common parameters are $k=0.2$ and $\lambda=0.4$.

as the exchanges of energy and water between the land and the atmosphere. Its stochastic dynamics, driven by random pulses of rainfall, is a clear example of a jump process, which has been quite extensively studied in the context of arid and semiarid ecohydrology $[14,40]$. In this section a simplified stochastic model for the soil water balance is presented and the effect of different distributions of precipitation event depths is analyzed.

\section{A. Model description}

Following the modeling scheme of $[14,22]$, the soil can be considered to be a constant storage capacity $w_{0}$; the daily soil water balance at a point can thus be schematically described by

$$
w_{0} \frac{d x}{d t}=-E_{T}(x)+I(x, t),
$$

where $x$ is the effective relative soil moisture defined between 0 (e.g., dry soil) and 1 (e.g., saturated soil), $E_{T}(x)$ is the evapotranspiration rate, and $I(x, t)$ is the infiltration rate, i.e., the amount of rainfall entering the soil. The water content is randomly forced by precipitation, which generates fast increments of soil moisture. We assume that the spatial scales considered are small enough for moisture recirculation to be negligible, so that rainfall events are independent of the soil moisture conditions. With this assumption, at the daily time scale and within a growing season, rainfall can be idealized as a compound Poisson process, whose events occur at a constant rate $\lambda$ and carry a random amount of water extracted from a distribution $h(y)$ with mean $\alpha_{r}$. When a rainfall event carries more water than the soil can accommodate, $x$ reaches the upper bound at 1 and the excess of water is lost through leakage and/or runoff $L$. In the other cases the whole rainfall amount infiltrates the soil. The infiltration process, which determines the increments of soil moisture following precipitation events, is thus related to that of rainfall and also depends on the value of $x(t)$ at the moment of the jump.

Between rainfall events, $x$ decreases because of evapotranspiration. Evapotranspiration is assumed to be a function of the water available to vegetation (i.e., $x$ ) and the potential evapotranspiration $E_{p}$, which is the maximum rate of evapo- transpiration achievable under well-watered conditions $(x=1)$. In the following, evapotranspiration rate $E_{T}(x)$ is assumed to decrease linearly from $E_{p}$ at $x=1$ to zero at $x=0$ [22]. Equation (37) can thus be written as

$$
\frac{d x}{d t}=-\eta x+F(x, t)
$$

where $\eta=E_{p} / w_{0}$ and $F(x, t)$ is the infiltration rate normalized with respect to $w_{0}$.

The dynamics of $x$ in steady-state conditions can be interpreted as the soil moisture dynamics during a growing season [22]. Therefore, the pdf $p(x)$ can be assumed to be representative of the soil moisture statistics for a period of several months (e.g., 6-7 months). Because we are focusing on the process at steady state, the presence of the bound at $x=1$ can be taken into account by renormalizing between 0 and 1 the pdf of the unbounded process (see Sec. II B).

In order to find $p(x)$, the distribution of the rainfall amount per event (i.e., the jumps) needs to be defined. The daily rainfall amount per event has been often assumed to be exponentially distributed $[14,22,24,26]$. This choice reduces the number of parameters involved in the model and allows exact mathematical derivation of the pdf of $x$ [22]. However, in some regions and for specific applications, daily rainfall depth may be better by more flexible distributions, such as the gamma distribution. For example, the shape parameter $\alpha$ of these gamma distributions has been observed to vary between 0.5 and 0.6 in monsoon regions of China and 1.2 in southern Mexico [41]. Moreover, although the exponential and the gamma distributions in many cases fit data for low values of rainfall amount per event, in other situations they may fail to fit the tail of the distributions, and mixtures of exponential distributions have been employed because of their heavier tails $[42,43]$.

Here, we compare outcomes of the model in Eq. (38) using three different types of distribution for daily rainfall with the same average $\alpha_{r}$. We will use an exponential distribution with parameter $\gamma=w_{0} / \alpha_{r}$; gamma distributions with $\alpha$ equal to $2,0.6,1.2$, and $\theta=\alpha w_{0} / \alpha_{r}$; and a mixture of exponential distributions for which $\left(\omega_{1} / \gamma_{1}\right)+\left[\left(1-\omega_{1}\right) / \gamma_{2}\right]$ $=\alpha_{r} / w_{0}$. 

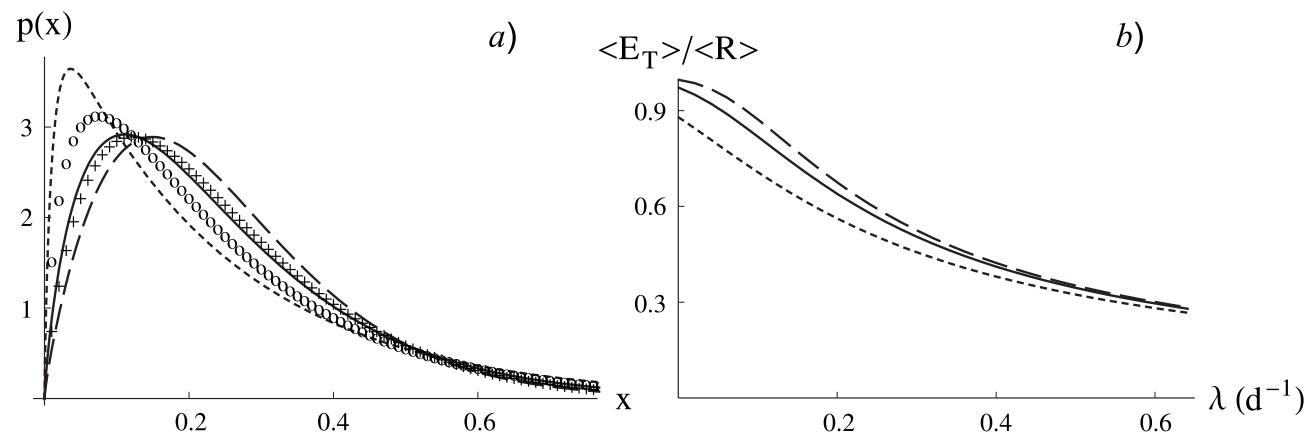

FIG. 4. (a) Different steady-state pdf's of $x\left(\lambda=0.1 \mathrm{~d}^{-1}\right)$ and (b) percentage of rainfall lost via evapotranspiration during a growing season for processes forced by jumps with different distributions: exponential (continuous line: $\left.\gamma=w_{0} / \alpha\right)$, gamma $\left(\theta=\alpha w_{0} / \alpha_{r} ;\right.$ dashed line: $\alpha=2 ;+: \alpha=1.2 ; \bigcirc: \alpha=0.6)$, and mixture of two exponential distributions $\left(\left(\omega_{1} / \gamma_{1}\right)+\left[\left(1-\omega_{1}\right) / \gamma_{2}\right]=\alpha_{r} / w_{0} ;\right.$ dotted line: $\omega_{1}=0.45, \gamma_{1}$ $\left.=10 w_{0} / \alpha_{r}\right)$. Common parameters are $w_{0}=7.2 \mathrm{~cm}, E_{p}=0.4 \mathrm{~cm} \mathrm{~d}^{-1}$, and $\alpha_{r}=1 \mathrm{~cm}$.

Figure 4 reports a comparison of the steady-state pdf of $x$ forced by precipitation differently distributed. The solutions with the rainfall amounts gamma distributed with parameter different from 2 have been numerically calculated using the numerical Laplace inversion package of MATHEMATICA [44], which has been tested against the exact available solutions.

Although the average amount of water carried by each rainfall event is the same in all the examples, the mean of $x$ for various daily rainfall distributions is different because of the presence of the bound at 1 . The pdf obtained with daily rainfall gamma distributed with $\alpha=1.2$ is very similar to that obtained with precipitation events exponentially distributed, as expected. As the parameter $\alpha$ decreases, the mode of the pdf of $x$ becomes lower than that of $p_{e}(x)$. Like in the unbounded case (see Fig. 2), the mixed exponential, depending on the values of the parameters, can result in $p_{m}(x)$ largely more positively skewed than $p_{e}(x)$ and $p_{g}(x)$.

\section{B. Average water balance}

When soil moisture reaches steady state, the average water balance (i.e., the seasonal water balance) can be written as

$$
\frac{\left\langle E_{T}\right\rangle}{\langle R\rangle}+\frac{\langle L\rangle}{\langle R\rangle}=1,
$$

where $\left\langle E_{T}\right\rangle,\langle R\rangle$, and $\langle L\rangle$ are the average evapotranspiration, rainfall, and leakage rates, respectively. According to the modeling scheme adopted, the balance can be written as

$$
\frac{E_{p}\langle x\rangle}{\lambda \alpha_{r}}+\frac{\nu_{1} \alpha_{l}}{\lambda \alpha_{r}}=1
$$

where $\nu_{1}=\left.\eta p(x)\right|_{x=1}$ is the frequency of occurrence of leakage events (i.e., the frequency of up-crossing the level $x=1$ ) and $\alpha_{l}$ is the average of the leakage events, which means the average of the portions of jumps above $1-x(t)$. Equation (40) hence allows us to calculate $\alpha_{l}$ once $p(x)$ is known. When the daily rainfall amount is exponentially distributed, the distribution of leakage has the same exponential distribution because of the memoryless property of the exponential distribution. In this case $\alpha_{l}=\alpha_{r}$, as it can be verified from Eq. (40).
Figure 4 shows the percentage of annual rainfall rate lost through evapotranspiration rate with different $h(y)$ 's. The largest differences in the partition of rainfall into evapotranspiration and leakage occur for low rainfall frequencies. In fact, in such a case the leakage strongly depends on the tail of the distribution of the jumps, and consequently the mixed exponential distribution generates the highest percentage of water lost via leakage because of its heavier tail.

\section{Level-crossing properties}

The differences in the statistical properties of $x$ caused by various distributions of the rainfall amount per event affect important mechanisms related to the soil water content. For example, as already mentioned, if soil moisture reaches saturation (i.e., $x=1$ ) after a rainfall event, water is lost because of percolation or runoff. Especially in arid and semiarid climates, it might be useful to estimate the averaged time of leakage and runoff occurrence, $T_{1^{-}}$, in order to define the water availability in streams. Such a variable has been used, for instance, as input for other models dealing with runoff and leakage estimations at small watershed scales [45]. As shown in Fig. 5, estimates of $T_{1^{-}}$are largely influenced by the distribution of the rainfall intensities especially for low rainfall frequencies.

The crossing properties of other soil moisture levels might be of interest in relation to, e.g., the vegetation water stress [22]. Again, as reported in Fig. 5 in the case of $T_{0.7^{-}}$, various distributions of daily rainfall amount per event lead to different estimates of the time spent below a certain level. These differences, as expected, become more marked as $\lambda$ decreases.

\section{CONCLUSIONS}

The paper studied the effect of different jump distributions on the dynamics of jump processes. General properties are reviewed and the case of systems with linear drift is analyzed in detail. Exact pdf's of the state variable $x$ are reported in the case of jumps exponentially distributed, and $p(x)$ 's in the cases of jumps gamma distributed and distributed as a mixture of exponential distributions are derived. Particularly, a solution for the steady-state pdf $p(x)$ when $x$ is 

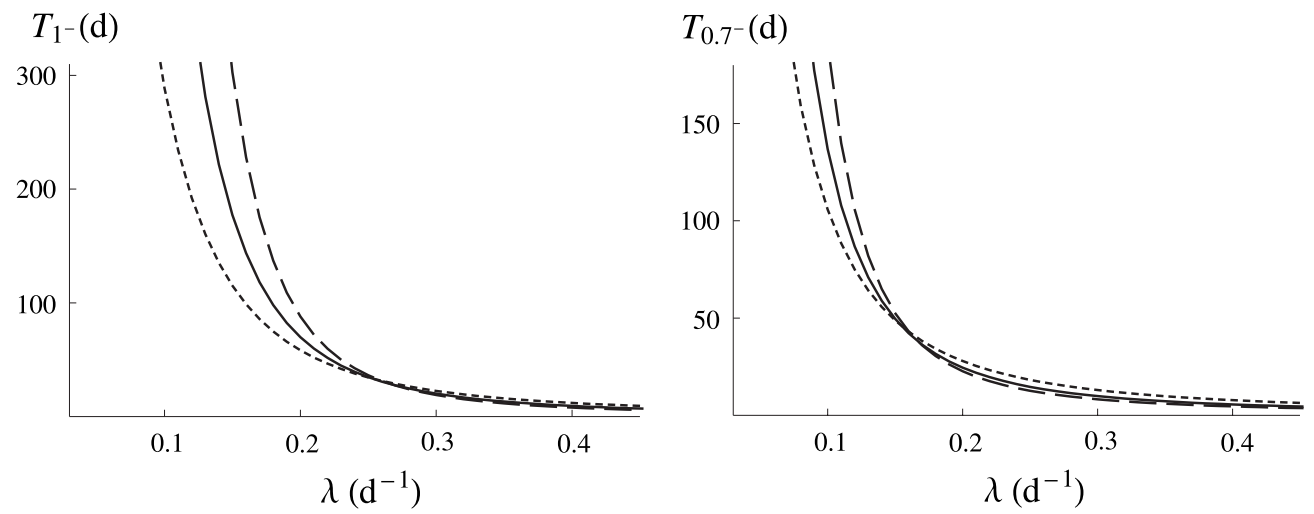

FIG. 5. Comparison between the average occurrence of runoff and the duration of an excursion below the level $\xi=0.7$ as a function of rainfall frequency for processes forced by jumps with different distributions: exponential (continuous line: $\gamma=w_{0} / \alpha$ ), gamma (dashed line: $\left.\alpha=2, \theta=\alpha w_{0} / \alpha_{r}\right)$, and mixture of two exponential distributions (dotted line: $\left.\omega_{1}=0.45, \gamma_{1}=10 w_{0} / \alpha_{r},\left(\omega_{1} / \gamma_{1}\right)+\left[\left(1-\omega_{1}\right) / \gamma_{2}\right]=\alpha_{r} / w_{0}\right)$. Common parameters are $n=0.4, Z_{r}=30 \mathrm{~cm}, w_{0}=7.2 \mathrm{~cm}, E_{p}=0.4 \mathrm{~cm} \mathrm{~d}^{-1}$, and $\alpha_{r}=1 \mathrm{~cm}$.

forced by jumps gamma distributed with shape parameter equal to 2 is derived [Eq. (30)], and an exact expression for $p(x)$ when the jumps are distributed as a mixture of two exponential distributions is presented [Eq. (35)]. These analytical solutions are used to study the effect of the jump distribution on the average duration of an excursion below a level $x=\xi$. As $\xi$ increases, these average times appear strongly reduced for jump distributions with heavier tails (Fig. 3).

The previous results are extended to bounded processes and adopted in a schematic model for the soil water balance, for which the state variable $x$ is bounded between 0 and 1 . The jumps in this model are due to daily rainfall event. The distribution of daily precipitation affects the statistics of the effective relative soil moisture (Fig. 4) as well as the crossing properties of thresholds associated with vegetation water stress and occurrence of leakage and runoff (Fig. 5). The results from this model also suggest that the choice of the distribution of daily rainfall depths is particularly important when the rainfall frequency is low. These low rainfall frequencies are characteristic of arid and semiarid climates and might well represent future climatic scenarios in some regions, for which rainfall is predicted to lower its frequency and to increase the water amount per event.

\section{APPENDIX A: FREQUENCY OF DOWN-CROSSING}

In steady-state conditions, the master equation reads

$$
\frac{d}{d x}[f(x) p(x)]-\lambda p(x)+\lambda \int_{-\infty}^{x} p(z) h(x-z) d z=0 .
$$

Integrating the previous equation with respect to $x$ between $-\infty$ and the level $\xi$ yields

$$
f(\xi) p(\xi)-\lambda \int_{-\infty}^{\xi} p(x) d x+\lambda \int_{-\infty}^{\xi}\left(\int_{-\infty}^{x} p(z) h(x-z) d z\right) d x=0 .
$$

The double integral can be rewritten as

$$
\begin{aligned}
\int_{-\infty}^{\xi}\left(\int_{-\infty}^{x} p(z) h(x-z) d z\right) d x & =\int_{-\infty}^{\xi} p(z)\left(\int_{z}^{\xi} h(x-z) d x\right) d z \\
& =\int_{-\infty}^{\xi} p(z)\left(\int_{0}^{\xi-z} h(y) d y\right) d z \\
& =\int_{-\infty}^{\xi}\left(1-\int_{\xi-z}^{+\infty} h(y) d y\right) p(z) d z
\end{aligned}
$$

which when inserted in Eq. (A2) leads to

$$
\nu_{\xi}=\lambda \int_{-\infty}^{\xi}\left(\int_{\xi-z}^{+\infty} h(y) d y\right) p(z) d z=f(\xi) p(\xi) .
$$

\section{APPENDIX B: DERIVATION OF $p(x, t)$ WITH LINEAR DRIFT}

When $f(x)=k x \quad(k>0)$, transforming Eq. (3) in the Laplace domain yields

$$
\frac{\partial}{\partial t} p^{*}(u, t)+k u \frac{\partial}{\partial u} p^{*}(u, t)=-\lambda\left[1-h^{*}(u)\right] p^{*}(u, t),
$$

with initial condition $p^{*}(u, 0)=\exp \left(-x_{0} u\right)$. Equation (B1) can be solved using the method of the characteristics. We look for two independent integrals $\phi\left(t, u, p^{*}\right)=C_{1}$ and $\psi\left(t, u, p^{*}\right)$ $=C_{2}$ of the characteristic system

$$
d t=\frac{d u}{k u}=\frac{d p^{*}}{-\lambda\left[1-h^{*}(u)\right] p^{*}} .
$$

The first integral can be found by solving $k d t=d u / u$ and reads

$$
C_{1}=u e^{-k t} .
$$

The second integral can be found by solving

$$
\frac{d p^{*}}{p^{*}}=\frac{-\lambda\left[1-h^{*}(u)\right]}{k u} d u,
$$

which gives 


$$
C_{2}=p^{*} \exp \left(\int_{u} \frac{\lambda\left[1-h^{*}\left(u^{\prime}\right)\right]}{k u^{\prime}} d u^{\prime}\right)
$$

The initial condition $p^{*}(u, 0)=\exp \left(-x_{0} u\right)$ can be written in parametric form as

$$
t=0, \quad u=\xi, \quad p^{*}=\exp \left(-x_{0} \xi\right)
$$

from which the two constants $C_{1}$ and $C_{2}$ result to be

$$
\begin{aligned}
C_{1} & =\xi=u e^{-k t} \\
C_{2} & =e^{-x_{0} \xi} \exp \left(\int_{\xi} \frac{\lambda\left[1-h^{*}\left(u^{\prime}\right)\right]}{k u^{\prime}} d u^{\prime}\right) \\
& =p^{*} \exp \left(\int_{u} \frac{\lambda\left[1-h^{*}\left(u^{\prime}\right)\right]}{k u^{\prime}} d u^{\prime}\right) .
\end{aligned}
$$

The function $p^{*}(u, t)$ can be explicitly derived by eliminating the parameter $\xi$ from system (B7) and it reads as Eq. (16).

\section{APPENDIX C: JUMP SIZE GAMMA DISTRIBUTED}

When $h(y)=\theta^{\alpha} y^{\alpha-1} e^{-\theta y} / \Gamma(\alpha)$, the function $h^{*}(u)$ reads

$$
h^{*}(u)=\frac{\theta^{\alpha}}{(\theta+u)^{\alpha}} .
$$

Equation (16) thus reads

$$
p_{g}^{*}(u, t)=e^{-x_{0} u e^{-k t}} \exp \left(\int_{u e^{-k t}}^{u} \frac{\left[\lambda \theta^{\alpha} /(\theta+s)^{\alpha}-\lambda\right]}{k s} d s\right)
$$

which gives

$$
p_{g}^{*}(u, t)=e^{-x_{0} u e^{-k t}-\lambda t} \exp \left(\frac{\lambda}{k} \int_{u e^{-k t}}^{u} \frac{\theta^{\alpha}}{(\theta+s)^{\alpha} s} d s\right) .
$$

The integral on the right-hand side of the previous equation can be written as

$$
\frac{\lambda}{k \theta} \int_{u e^{-k t}}^{u}(-1)^{\alpha+1}(-\theta / s)^{\alpha+1}(1+\theta / s)^{-\alpha} d s,
$$

which, by using Eq. (15.1.8) of [25], can be rewritten in terms of the hypergeometric function $F(\cdot, \cdot ; \cdot ; \cdot)$ as

$$
\frac{\lambda}{k \theta} \int_{u e^{-k t}}^{u}(-1)^{\alpha+1}(-\theta / s)^{\alpha+1} F(\alpha, \alpha ; \alpha ;-\theta / s) d s,
$$

which can be solved as (Eq. (15.2.4) of [25])

$$
-\left.\frac{\lambda}{k \alpha}\left(\frac{\theta}{s}\right)^{\alpha} F(\alpha, \alpha ; \alpha+1 ;-\theta / s)\right|_{u e^{-k t}} ^{u} .
$$

Equation (26) is obtained by inserting the previous equation into Eq. (C3).

\section{APPENDIX D: JUMP SIZE DISTRIBUTED AS A MIXTURE OF TWO EXPONENTIAL DISTRIBUTIONS}

When $h(y)=\omega_{1} \gamma_{1} \exp \left(-\gamma_{1} y\right)+\left(1-\omega_{1}\right) \gamma_{2} \exp \left(-\gamma_{2} y\right), p(x)$ is given by the convolution of two gamma distributions defined in Eq. (34). Accordingly,

$$
\begin{aligned}
p_{m}(x)= & \frac{\gamma_{1}^{\lambda \omega_{1} / k} \gamma_{2}^{\lambda\left(1-\omega_{1}\right) / k}}{\Gamma\left(\lambda \omega_{1} / k\right) \Gamma\left(\lambda\left[1-\omega_{1}\right] / k\right)} \int_{0}^{x} e^{-\gamma_{1} z^{-1+\lambda \omega_{1} / k} e^{-\gamma_{2}(x-z)}} \\
& \times(x-z)^{-1+\lambda\left(1-\omega_{1}\right) / k} d z .
\end{aligned}
$$

By operating the variable change $z=s x$ and rearranging the terms, the previous equation can be written as

$$
\begin{aligned}
p_{m}(x)= & \frac{\gamma_{1}^{\lambda \omega_{1} / k} \gamma_{2}^{\lambda\left(1-\omega_{1}\right) / k} e^{-\gamma_{2} x} x^{-1+\lambda / k}}{\Gamma\left(\lambda \omega_{1} / k\right) \Gamma\left(\lambda\left[1-\omega_{1}\right] / k\right)} \int_{0}^{1} e^{-\left(\gamma_{1}-\gamma_{2}\right) x s} s^{-1+\lambda \omega_{1} / k} \\
& \times(1-s)^{-1+\lambda\left(1-\omega_{1}\right) / k} d s
\end{aligned}
$$

which according to Eqs. (13.2.1) of [25] can be re-written as

$$
\begin{aligned}
p_{m}(x)= & \frac{e^{-\gamma_{2} x} \gamma_{1}^{\lambda \omega_{1} / k} \gamma_{2}^{\lambda\left(1-\omega_{1}\right) / k} x^{-1+\lambda / k}}{\Gamma(\lambda / k)} \\
& \times_{1} F_{1}\left(\frac{\lambda \omega_{1}}{k}, \frac{\lambda}{k},-\left(\gamma_{1}-\gamma_{2}\right) x\right),
\end{aligned}
$$

which equals Eq. (35) when Eq. (13.1.27) of [25] is applied.
[1] E. Parzen, Stochastic Processes (Holden-Day Inc., San Francisco, 1964).

[2] O. Kella, D. Perry, and W. Stadje, Probability in the Engineering and Informational Sciences 17, 1 (2003).

[3] H. Askari and N. Krichene, Energy Economics 30, 2134 (2008).

[4] H. C. Tuckwell, Introduction to Theoretical Neurobiology. II. Nonlinear and Stochastic Theories (Cambridge University Press, Cambridge, England, 1988).

[5] M. J. Chacron, B. Lindner, and A. Longtin, Phys. Rev. Lett. 92, 080601 (2004).

[6] B. Lindner, M. J. Chacron, and A. Longtin, Phys. Rev. E 72, 021911 (2005).
[7] E. Daly and A. Porporato, Phys. Rev. E 75, 011119 (2007).

[8] F. B. Hanson and H. C. Tuckwell, Theor Popul. Biol. 19, 1 (1981).

[9] M. Abundo, Open Syst. Inf. Dyn. 11, 105 (2004).

[10] Y. Wu and W. Q. Zhu, Phys. Rev. E 77, 041911 (2008).

[11] J. S. Clark, Oikos 56, 17 (1989).

[12] P. D'Odorico, F. Laio, and L. Ridolfi, Am. Nat. 167, E79 (2006).

[13] G. Weiss, Water Resour. Res. 13, 101 (1977).

[14] I. Rodriguez-Iturbe, A. Porporato, L. Ridolfi, V. Isham, and D. R. Cox, Proc. R. Soc. London, Ser. A 455, 3789 (1999).

[15] J. Luczka, R. Bartussek, and P. Hänggi, EPL 31, 431 (1995).

[16] H. Nakao, K. S. Arai, K. Nagai, Y. Tsubo, and Y. Kuramoto, 
Phys. Rev. E 72, 026220 (2005).

[17] K. Arai and H. Nakao, Phys. Rev. E 77, 036218 (2008).

[18] E. Daly and A. Porporato, Physica D 238, 170 (2009).

[19] J. Candia, M. C. Gonzales, P. Wang, T. Schoenharl, G. Madey, and A.-L. Barabasi, J. Phys. A: Math. Theor. 41, 224015 (2008).

[20] M. S. Wheatland, Astrophys. J. 679, 1621 (2008).

[21] M. S. Wheatland, Sol. Phys. 255, 211 (2009).

[22] A. Porporato, E. Daly, and I. Rodriguez-Iturbe, Am. Nat. 164, 625 (2004).

[23] D. R. Cox and H. D. Miller, The Theory of Stochastic Processes (Methuen, London, 1965).

[24] E. Daly and A. Porporato, Water Resour. Res. 42, W06401 (2006).

[25] Handbook of Mathematical Functions, edited by M. Abramowitz and I. A. Stegun (Dover, New York, 1965).

[26] E. Daly and A. Porporato, Phys. Rev. E 73, 026108 (2006).

[27] I. F. Blake and W. C. Lindsey, IEEE Trans. Inf. Theory 19, 295 (1973).

[28] A. Porporato, F. Laio, L. Ridolfi, and I. Rodriguez-Iturbe, Adv. Water Resour. 24, 725 (2001).

[29] F. Laio, A. Porporato, L. Ridolfi, and I. Rodriguez-Iturbe, Phys. Rev. E 63, 036105 (2001).

[30] K. Borovkov and G. Last, Adv. Appl. Probab. 40, 815 (2008).

[31] M. R. Leadbetter and G. V. Spaniolo, Aust. N. Z. J. Stat. 46, 173 (2004).
[32] A. Novikov, R. E. Melchers, E. Shinjikashvili, and N. Kordzakhia, Probab. Eng. Mech. 20, 57 (2005).

[33] L. Takács, Proceedings of the Fourth Berkeley Symposium on Mathematical Statistics and Probability (Univ. of California Press, Berkeley, 1961), Vol. 2, pp. 535-567.

[34] S. I. Denisov, W. Horsthemke, and P. Hänngi, Eur. Phys. J. B 68, 567 (2009).

[35] A. P. Prudnikov, Y. A. Brychkov, and O. I. Marichev, Integrals and Series (Gordon and Breach, New York, 1986), Vol. 5.

[36] A. Tsurui and S. Osaki, Stochastic Proc. Appl. 4, 79 (1976).

[37] F. Viola, E. Daly, G. Vico, M. Cannarozzo, and A. Porporato, Water Resour. Res. 44, W11412 (2008).

[38] O. Kella and W. Stadje, J. Appl. Probab. 38, 781 (2001).

[39] A. Feldmann and W. Whitt, Perform. Eval. 31, 245 (1998).

[40] I. Rodriguez-Iturbe and A. Porporato, Ecohydrology of WaterControlled Ecosystems: Soil Moisture and Plant Dynamics (Cambridge University Press, Cambridge, England, 2004).

[41] P. Y. Groisman et al., Clim. Change 42, 243 (1999).

[42] E. M. Furrer and R. W. Katz, Water Resour. Res. 44, W12439 (2008).

[43] E. Foufoula-Georgiou and D. P. Lettenmaier, Water Resour. Res. 23, 875 (1987).

[44] P. P. Valkó and J. Abate, Comput. Math. Appl. 48, 629 (2004) (http://library.wolfram.com/infocenter/MathSource/4738/).

[45] G. Botter, A. Porporato, E. Daly, I. Rodriguez-Iturbe, and A. Rinaldo, Water Resour. Res. 43, W06404 (2007). 